\title{
Grip and load force coordination in cyclical isometric manipulation task is not affected by the feedback type
}

\author{
Sabrina Tiago Pedão ${ }^{1}$, José Angelo Barela ${ }^{1,2}$, Kauê Carvalho de Almeida Lima ${ }^{1}$ and Paulo Barbosa de Freitas ${ }^{1 *}$
}

\begin{abstract}
Background: The relationship between normal and tangential force components (grip force - GF and load force - LF, respectively) acting on the digits-object interface during object manipulation reveals neural mechanisms involved in movement control. Here, we examined whether the feedback type provided to the participants during exertion of LF would influence GF-LF coordination and task performance.

Methods: Sixteen young ( $24.7 \pm 3.8$ years-old) volunteers isometrically exerted continuously sinusoidal $F_{z}$ (vertical component of LF) by pulling a fixed instrumented handle up and relaxing under two feedback conditions: targeting and tracking. In targeting condition, $F_{Z}$ exertion range was determined by horizontal lines representing the upper $(10 \mathrm{~N})$ and lower $(1 \mathrm{~N})$ targets, with frequency $(0.77$ or $1.53 \mathrm{~Hz})$ dictated by a metronome. In tracking condition, a sinusoidal template set at similar frequencies and range was presented and should be superposed by the participants' exerted $F_{Z}$. Task performance was assessed by absolute errors at peaks (AE $E_{\text {peak }}$ ) and valleys ( $A E_{\text {Valley }}$ ) and GF-LF coordination by GF-LF ratios, maximum cross-correlation coefficients ( $\left.r_{\max }\right)$, and time lags.

Results: The results revealed no effect of feedback and no feedback by frequency interaction on any variable. $A E_{\text {peak }}$ and GF-LF ratio were higher and $r_{\text {max }}$ lower at $1.53 \mathrm{~Hz}$ than at $0.77 \mathrm{~Hz}$.

Conclusion: These findings indicate that the type of feedback does not influence task performance and GF-LF coordination. Therefore, we recommend the use of tracking tasks when assessing GF-LF coordination during isometric LF exertion in externally fixed instrumented handles because they are easier to understand and provide additional indices (e.g., RMSE) of voluntary force control.
\end{abstract}

Keywords: Visual, Auditory, Hand function, Motor control

\section{Background}

The ability to use one or both hands to grasp and manipulate objects is essential for performing innumerous daily living activities and, consequently, is important for maintaining an independent lifestyle. Therefore, several studies have explored different aspects of hand function. Specifically, a largely used and elegant experimental paradigm has been applied to investigate the relationship between force components acting on the digits and object surface interface during object manipulation. The force component acting tangentially on the digits-object

\footnotetext{
* Correspondence: deFreitasPB@gmail.com

'Institute of Physical Activity and Sport Sciences and Graduate Program in Human Movement Sciences, Cruzeiro do Sul University, Rua Galvão Bueno $868,13^{\circ}$ andar, Bloco B, São Paulo 01506-000, SP, Brazil

Full list of author information is available at the end of the article
}

interface, referred to as load force (LF), tends to cause slippage of the handheld object, which is prevented by the exertion of force perpendicularly to the object surface, which has been termed grip force (GF) [1-3].

The close relationship established between GF and LF during object manipulation is a striking evidence of the central nervous system's (CNS) ability to predict the effects of individuals' own actions [2,4-6]. This coupling has been widely investigated during manipulation tasks that involve lifting a grasped object [1], moving a handheld object upward and downward discretely [7] or continuously $[5,8]$, and isometrically applying sinusoidal LF profiles on an externally fixed object $[4,6,9]$. This highly coupled relationship is characterized by a parallel change of GF and LF with virtually no time delay. During tasks in

\section{Ciomed Central}


which LF changes (e.g., lifting, transporting, and shaking a handheld object) this parallel change of GF and LF ensure an economical exertion of GF $[1,4,5,7,9]$.

Investigations of GF-LF coupling using continuous changes in LF during manipulation of free moving and fixed objects have provided consistent support for the use of predictive strategies by CNS in healthy $[4,5]$ and neurological individuals [10-12]. However, the use of an externally fixed object could be advantageous when compared with the use of a free moving one because researchers could easily regulate LF magnitude and frequency. Also, additional information about the CNS current condition and about the adopted control strategies related to visuomotor coordination could be obtained by assessing the individual's ability to control LF (i.e., task performance) over the task time course. Hence, a common methodological feature of this type of experiment is to provide both the real time visual feedback of the exerted LF and any type of information regarding the prescribed LF magnitude and frequency to guide participants in the task execution. For example, in two studies performed in fixed (or quasi-fixed) objects, visual information about the prescribed LF magnitude and frequency and about the currently exerted LF was presented in oscilloscopes and computer screens $[4,10,13]$. Moreover, in other studies [6,9], visual information about the prescribed LF magnitude was shown in a computer screen (i.e., visual feedback) while information about LF frequency was given by a metronome (auditory feedback).

To our knowledge, no one compared the effects of exerting cyclical isometric LF using different types of feedback on task performance as well as on GF scaling and GF-LF coupling. The comparison between two different types of feedback (visual vs. visual plus auditory) is a relevant methodological aspect when designing studies aiming to investigate the relationship between GF and LF and to assess hand function in healthy and hand-impaired individuals. Regarding task performance, we could expect differences between feedback conditions because any motor task that requires simultaneous processing of visual and auditory feedback could be considered more complex than a task that requires processing of a single source of feedback because individuals need to deal with two different sources of information, which increases, for example, attentional demands. Thus, looking from this perspective, individuals with attention deficits (e.g., old adults, Parkinson and cerebellar patients, and specific group of children) would benefit from a simplified experimental approach as the proposed by this study. Conversely, regarding GF scaling and GF-LF coupling, the need for accuracy throughout the whole task imposed by a visual task could be a burden on the CNS and it would make the system to change the adopted control strategy, that is, increase GF magnitude and reduce GF-LF coupling.
Thus, before recommending one experimental approach in detriment of another we need to investigate whether they produce different outcomes in variables related to GF-LF coordination as well as task performance. Therefore, we examine in this study whether the type of feedback received by the individuals during exertion of oscillatory isometric LF profile would influence task performance and GF scaling, as well as GF-LF coordination. If we find differences in task performance and, mainly, in GF-LF coordination we would have to take into consideration this fact before selecting one type of feedback that interfere less with GF-LF coordination. Alternatively, if we find no differences in those variable we could claim that the type of feedback received by the participants is not influential in GF-LF coordination and could recommend the use of either one based upon the advantages that one type of feedback has comparing to the other.

\section{Methods \\ Participants}

Sixteen healthy and right-handed individuals (eight males), ranging from 19 to 33 years of age (mean \pm S.D.: $24.7 \pm 3.8$ years-old) participated in the study. Their participation was conditioned to the signature of an informed consent form. All experimental procedures and the informed consent form given to and signed by the participants were approved by the research ethics committee of the Cruzeiro do Sul University. This study was conducted in accordance with the Declaration of Helsinki.

\section{Instrumentation}

An instrumented handle consisting of two parallel aluminum plates $(15 \times 4 \mathrm{~cm})$ connected with each other by a single-axis force transducer (LPM-530, Cooper Instruments and Systems, USA) and two aluminum pieces with a multiaxis force and torque (F/T) transducer (Mini40, ATI, USA) in between them (forming the base of the handle) was used in the study (Figure 1A). The opposing handle grasping surfaces were $5 \mathrm{~cm}$ apart and were covered with sandpaper (320 grit). The single-axis force transducer recorded the compression force $\left(\mathrm{F}_{\mathrm{C}}\right)$ exerted by the tip of the thumb against the handle, while the F/T transducer recorded all three force and torque components applied against the handle by the digits. The horizontal force components acting perpendicularly to the handle contact areas (i.e., $\mathrm{F}_{\mathrm{C}}$ recorded by the single-axis and $\mathrm{F}_{\mathrm{Y}}$ recorded by the multi-axis $\mathrm{F} / \mathrm{T}$ transducer) were used to calculate the grip force $\left[G F=\left(F_{C}+\left(|| F_{C}-F_{Y}||\right)\right) / 2\right]$, i.e., the average force exerted against the two sides of the handle $[9,14]$. The vertical $\left(\mathrm{F}_{\mathrm{Z}}\right)$ and horizontal $\left(\mathrm{F}_{\mathrm{X}}\right)$ force components recorded by the $\mathrm{F} / \mathrm{T}$ transducer, both tangential to the handle surface, were used to calculate load force $\left[\mathrm{LF}=\sqrt{ }\left(\mathrm{F}_{\mathrm{Z}}^{2}+\mathrm{F}_{\mathrm{X}}^{2}\right)\right]$. 

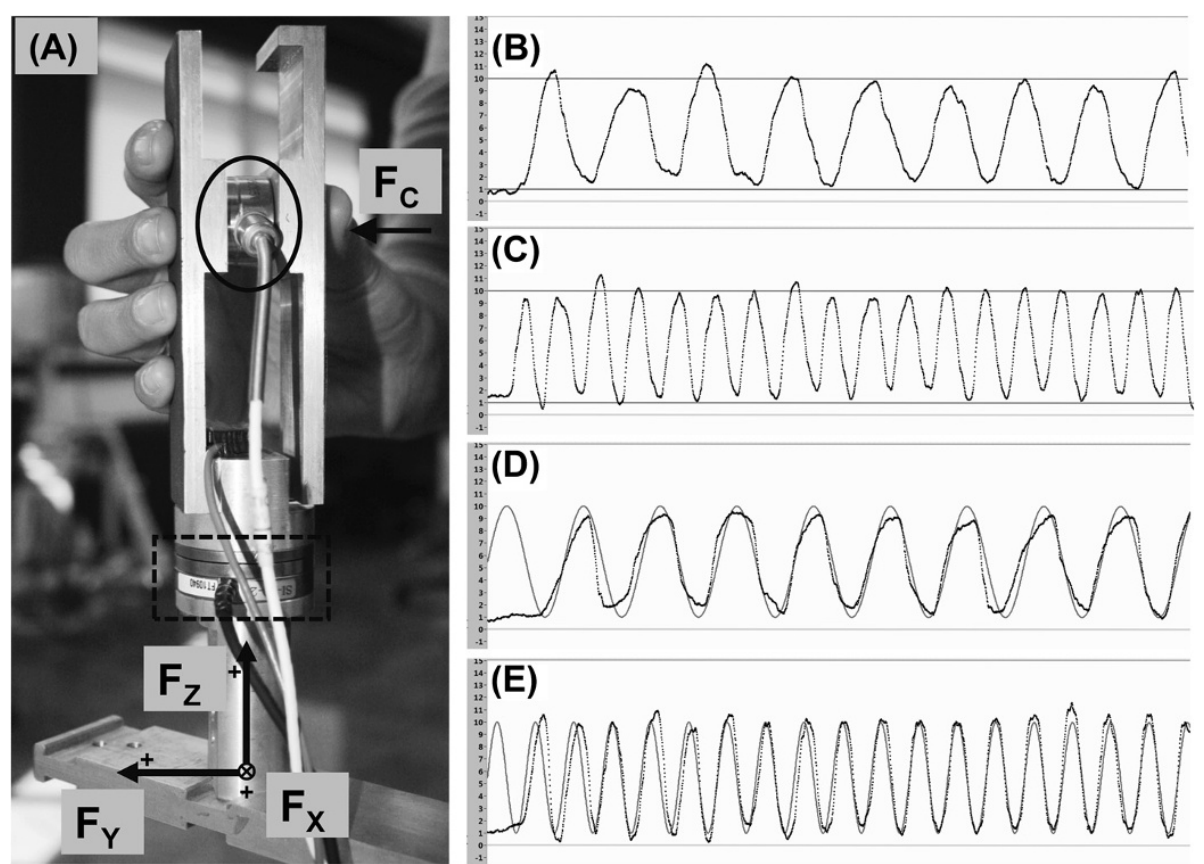

Figure 1 Instrumented handle photography and representation of all four experimental conditions. (A) Photography of the handle used in the experiment and representation of the force components recorded by the single-axis (ellipse) and multi-axis $F / T$ transducers (dashed rectangle). (B-E) The vertical force component of $L F\left(F_{Z}\right)$ time-series (black lines) of trials performed at $0.77 \mathrm{~Hz}(\mathbf{B}$ and $\mathbf{D})$ and at $1.53 \mathrm{~Hz}(\mathbf{C}$ and $\mathbf{E})$ in the targeting condition (B and $\mathbf{C}$ ), in which two horizontal lines (gray lines) displayed in the computer monitor represented the prescribed upper and lower targets, and in the tracking condition ( $\mathbf{D}$ and $\mathbf{E}$ ), in which a sinusoidal template (gray line) shown in the computer monitor represented the prescribed target.

\section{Experimental procedure}

Before starting the experiment, all participants cleaned the tip of their digits with alcohol swabs. Then, they stood upright, kept their upper arm vertically and forearm horizontally oriented, and were asked to grasp the fixed handle using the tip of their digits as shown in Figure 1A. The vertically oriented handle was rotated $45^{\circ}$ with respect to the participants' frontal plane to assure a comfortable wrist position. Next, the participants were asked to exert a sinusoidal pattern of the vertical component of LF $\left(\mathrm{F}_{\mathrm{Z}}\right)$, by isometrically pulling the handle up and relaxing up to a certain point. They were requested to do it within a prescribed range (i.e., $9 \mathrm{~N}$, ranging from 1 to $10 \mathrm{~N})$ at two different frequencies [46 bpm $(\approx 0.77 \mathrm{~Hz})$ and $92 \mathrm{bpm}(\approx 1.53 \mathrm{~Hz})$ ].

The participants performed the task receiving two distinct types of feedback: targeting and tracking. In the targeting condition, they received information of the prescribed $\mathrm{F}_{\mathrm{Z}}$ magnitude by two horizontal lines presented in a 19-in. widescreen computer monitor placed in front of them (Figure 1B-C) and information of the prescribed frequency by a metronome set at the above mentioned frequencies. They also received information about the current real-time $\mathrm{F}_{\mathrm{Z}}$ exerted by them shown as a continuous left to right running black line. The participants were instructed to continuously exert sinusoidal
$\mathrm{F}_{\mathrm{Z}}$ reaching the upper and lower targets (red lines) as accurate as possible following the rhythm dictated by the metronome. In the tracking condition, sinusoidal templates set at similar frequencies (either $0.77 \mathrm{~Hz}$ or $1.53 \mathrm{~Hz})$ and range $(9 \mathrm{~N}$, from 1 to $10 \mathrm{~N})$ were individually shown in the screen (Figure $1 \mathrm{D}-\mathrm{E}$ ). In this condition, the participants were asked to exert oscillatory $F_{Z}$ in order to match (i.e., superpose) the sinusoidal template, also as accurate as possible. The force range was selected based upon the fact that healthy young individuals, in the most of the daily situations in which they manipulate objects with a precision grip, do not generate high levels of tangential force due to the limitation of GF muscles to generate high magnitudes of grip to prevent slippage. Regarding the chosen frequencies, we selected a slow and a fast rate of $F_{Z}$ change, which allowed for a "within cycle" and a "next cycle" $\mathrm{F}_{\mathrm{Z}}$ corrections, respectively. Moreover, we chose $46 \mathrm{bpm}(\approx 0.77 \mathrm{~Hz})$ and $92 \mathrm{bpm}$ $(\approx 1.53 \mathrm{~Hz})$ because the commercially available metronomes have a multiple of two divisions starting at $40 \mathrm{bpm}$.

A period of familiarization with the apparatus, magnitude, and frequency was given to the participants. Namely, the participants performed between 2 and 5 trials to be able to perform the task properly, reaching the upper and lower targets and/or tracking accurately the sinusoidal profile. After, force signals from three trials for each 
condition of feedback and frequency were recorded at 200 $\mathrm{Hz}$ and stored for further analysis. Individual trials lasted $12 \mathrm{~s}$ and were performed in a balanced sequence, with half of the participants starting with the targeting condition and half starting with the tracking one. The same was done for frequency. Note that participants were only instructed to exert $\mathrm{F}_{\mathrm{Z}}$ by isometrically pulling up the handle and GF was not mentioned whatsoever.

\section{Data processing and analyses}

Two customized LabView (Version 2010, National Instruments, Austin, TX, USA) routines were used for data acquisition and processing. The raw force data were lowpass filtered at $20 \mathrm{~Hz}$ with a fourth-order (zero-phase lag) Butterworth filter. Data from the first $4 \mathrm{~s}$ (phase for $\mathrm{F}_{\mathrm{Z}}$ frequency and magnitude adjusts) and the last $2 \mathrm{~s}$ (end of trial) of each trial were not considered and removed after filtering processing. Therefore, only the data between the 4 th and 10th s were analyzed. Next, dependent variables related to task performance, GF scaling, and GF-LF coupling were calculated. Task performance was assessed by absolute errors (AE) [15] of $\mathrm{F}_{\mathrm{Z}}$ peaks and valleys with respect to the upper $\left(\mathrm{AE}_{\text {Peak }}\right)$ and lower $\left(\mathrm{AE}_{\mathrm{Valley}}\right)$ targets, respectively. GF-LF coordination was evaluated by measures of GF scaling and GF-LF coupling. GF scaling, which represents the CNS ability to scale GF with respect to LF, was assessed by GF-LF ratio (GF/LF), calculated as the averaged GF divided by the averaged absolute LF. Also, the maximum cross-correlation coefficient $\left(\mathrm{r}_{\max }\right)$ between GF and LF and its respective time lag (positive values indicating that GF lags LF) were computed from a linear cross-correlation analysis to assess, respectively, the directional and temporal coupling between LF and GF.

\section{Statistical analyses}

Five two-way, repeated measures, analyses of variance (ANOVA) were employed to test the effects of feedback (targeting vs. tracking) and frequency of $\mathrm{F}_{\mathrm{Z}}$ exertion $(0.77$ vs. $1.53 \mathrm{~Hz})$ and the interaction between these factors on $\mathrm{AE}_{\text {Peak }}, \mathrm{AE}_{\text {Valley }}$ (GF/LF), Fisher's $\mathrm{z}$ transformed of the $\mathrm{r}_{\max }$, and time lag. The significance level was set at .05.

\section{Results}

Overall, the results showed that the participants were able to exert sinusoidal $F_{Z}$ profiles reaching the lower and upper targets accurately (AEs in average less than $1 \mathrm{~N}$ ) at the requested frequencies independently of the type of feedback presented. Also, very high $r_{\max }$ values (>.87) with most of time lags ranging within $\pm 20 \mathrm{~ms}$ interval were observed regardless of feedback type and $F_{Z}$ frequency. The averaged values of the dependent variables related to task performance $\left(\mathrm{AE}_{\text {Peak }}\right.$ and $\left.\mathrm{AE}_{\text {Valley }}\right)$, GF scaling (GF/LF) and directional and temporal GF-LF coupling $\left(\mathrm{r}_{\max }\right.$ and time lag) are presented in Table 1.

\section{Task performance}

Regarding the effect of feedback and frequency on $\mathrm{AE}_{\text {Peak }}$, ANOVA revealed no main effect of feedback $[\mathrm{F}(1,15)=$ $\left.1.5, \mathrm{p}>.05, \eta^{2}=.09\right]$ and no feedback by frequency interaction $\left[F(1,15)=0.15, \mathrm{p}>.05, \eta^{2}=.03\right]$, but revealed that $\mathrm{AE}_{\text {Peak }}$ was larger at $1.53 \mathrm{~Hz}$ than at $0.77 \mathrm{~Hz}[\mathrm{~F}(1,15)=$ $\left.10.68, \mathrm{p}<.01, \eta^{2}=.42\right]$. For $\mathrm{AE}_{\mathrm{Valley}}$, however, ANOVA revealed neither main effect of feedback $[F(1,15)=2.5$, $\left.\mathrm{p}>.05, \eta^{2}=.14\right]$, nor of frequency $[\mathrm{F}(1,15)=0.56, \mathrm{p}>.05$, $\left.\eta^{2}=.04\right]$, and no feedback by frequency interaction $\left[F(1,15)=0.04, p>.05, \eta^{2}=.01\right]$.

\section{GF scaling}

ANOVA for GF/LF revealed neither effect of feedback $\left[F(1,15)=0.15, p>.05, \eta^{2}=.01\right]$ nor feedback by frequency interaction $\left[\mathrm{F}(1,15)=2.83, \mathrm{p}>.05, \eta^{2}=.16\right]$, but revealed that GF-LF ratio was higher at $1.53 \mathrm{~Hz}$ than at $0.77 \mathrm{~Hz}\left[\mathrm{~F}(1,15)=18.04, \mathrm{p}<.005, \eta^{2}=.55\right]$.

\section{Directional and temporal GF-LF coupling}

ANOVA for $r_{\max }$ Fisher's $\mathrm{z}$ transformed values revealed no main effect of feedback $\left[F(1,15)=0.4, p>.05, \eta^{2}=.03\right]$ and no feedback by frequency interaction $[\mathrm{F}(1,15)=0.005$, $\left.\mathrm{p}>.05, \eta^{2}=.01\right]$, but revealed that $\mathrm{r}_{\max }$ values were the

Table 1 Values representing task performance $\left(A E_{\text {Peak }}\right.$ and $\left.A E_{\text {Valley }}\right)$, GF scaling (GF/LF), and GF-LF coupling ( $r_{\text {max }}$ and time lag) in targeting and tracking feedback conditions performed at $0.77 \mathrm{~Hz}$ and $1.53 \mathrm{~Hz}$

\begin{tabular}{|c|c|c|c|c|c|c|}
\hline & & $A E_{\text {Peak }}(\mathrm{N})$ & $A E_{\text {Valley }}(\mathrm{N})$ & GF/LF & Time lag (ms) & $r_{\max }$ \\
\hline \multirow{4}{*}{ Targeting } & $0.77 \mathrm{~Hz}$ & $0.653^{b}$ & 0.596 & $0.779^{b}$ & 4.89 & $0.974^{a}$ \\
\hline & & $(0.077)$ & $(0.074)$ & $(0.055)$ & $(3.25)$ & \\
\hline & $1.53 \mathrm{~Hz}$ & $0.753^{\mathrm{a}}$ & 0.552 & $0.882^{a}$ & 9.27 & $0.962^{b}$ \\
\hline & & $(0.051)$ & $(0.056)$ & $(0.046)$ & $(4.42)$ & \\
\hline \multirow{4}{*}{ Tracking } & $0.77 \mathrm{~Hz}$ & $0.573^{b}$ & 0.491 & $0.738^{\mathrm{b}}$ & 6.04 & $0.98^{a}$ \\
\hline & & $(0.041)$ & $(0.058)$ & $(0.053)$ & $(3.17)$ & \\
\hline & $1.53 \mathrm{~Hz}$ & $0.733^{\mathrm{a}}$ & 0.461 & $0.95^{\mathrm{a}}$ & 9.22 & $0.947^{b}$ \\
\hline & & $(0.052)$ & $(0.056)$ & $(0.06)$ & (3.46) & \\
\hline
\end{tabular}


highest at the lower frequency (i.e., $0.77 \mathrm{~Hz})[\mathrm{F}(1,15)=$ 14.3, $\mathrm{p}<.005, \eta^{2}=.49$ ]. Regarding the time lag, ANOVA revealed no main effect of feedback $[\mathrm{F}(1,15)=0.71, \mathrm{p}>.05$, $\left.\eta^{2}=.05\right]$ and frequency $\left[\mathrm{F}(1,15)=1.04, \mathrm{p}>.05, \eta^{2}=.09\right]$, and no feedback by frequency interaction $[\mathrm{F}(1,15)=0.86$, $\left.\mathrm{p}>.05, \eta^{2}=.05\right]$.

\section{Discussion}

The aim of the study was to examine whether the type of feedback received by the participants while exerting oscillatory isometric LF profile would influence task performance and GF-LF coordination. The results showed that the type of feedback received did not impact any of the variables and that it is not frequency dependent. Moreover, the results revealed the already known effect of frequency on GF-LF coordination and task performance (lower $r_{\max }$ and higher GF-LF ratio, and higher $\mathrm{AE}_{\text {Peak }}$ at the higher frequencies) $[6,14]$.

Based upon these findings, someone would have no restrictions when selecting any of the feedback types tested in this study. However, the use of a tracking task could be advantageous when compared to the targeting one. Firstly, while in the targeting condition individuals need to deal with two sources of information (visual and auditory), in the tracking condition information about the prescribed LF magnitude and about LF frequency is presented simultaneously as a single entity (i.e., a sinusoidal template). Therefore, tracking would make the task easier because attention resources would be focused on a single source and not diverged in two as in a targeting task. Moreover, the tracking task would provide better guidance for task performance because, intuitively, it would be easier for children, older adults, and persons with mild neurological and orthopedic impairment to follow the instructions of tracking (i.e., superposing) a sinusoidal template than to reach the upper and lower targets during exerting LF in a sinusoidal manner following the beats of a metronome. Likewise, individuals who undergo hearing problems (e.g., old adults) could also be able to be tested when performing a tracking task. Secondly, a tracking task could provide additional and more complete estimation of task performance (i.e., force control) than a targeting one. Dependent variables such as absolute, variable and constant errors [15], related to LF peaks and valleys, could be calculated from both tasks. However, the ability to control force throughout the task execution could only be assessed in the tracking task by using, for instance, the root mean square error (RMSE) as a dependent variable.

Alternatively, someone could suggest the use of simple vertically moving horizontal bar that would oscillate upward and downward as the source of feedback about the prescribed frequency and amplitude [10] or the use of a task that involve pursuit of a sinusoidal moving target [16]. However, while those two feedback strategies could be as effective as the tracking task to provide visual guidance, they do not provide information about the continuing force path what could be useful for force magnitude and frequency corrections during task execution [17]. Also, information about force magnitude provided by a relatively rapid moving bar does not allow individual to give priority to a high level of accuracy.

There are limitations in this study that deserve to be mentioned and could be addressed in future investigations. For instance, participants exerted sinusoidal LF profile in only one force range $(1-10 \mathrm{~N})$. Despite Uygur and colleagues [14] having found no effect of LF magnitude in GF-LF coordination in LF ranging from 6 to $15 \mathrm{~N}$ during a task identical to the performed in targeting condition, the tracking task was not tested in different load conditions and the GF-LF coordination and task performance could be affected in higher and even lower LF amplitudes. In addition, we tested the participants in only two frequencies $(0.77$ and $1.53 \mathrm{~Hz}$ ). During isometric LF exertion in targeting condition, Jaric and colleagues [6] found that GF-LF coupling and GF scaling is affected in relatively very high frequency (above $3 \mathrm{~Hz}$ ). In frequencies lower than $2.5 \mathrm{~Hz}$, the indices of GF-LF coordination are kept relatively stable. However, those findings could not be applied to tracking conditions. Despite we observed no difference between feedback condition at $0.77 \mathrm{~Hz}$ and $1.53 \mathrm{~Hz}$ in GF scaling and GF-LF coupling, we could not affirm that feedback condition would not affect those parameters in frequencies higher than $1.53 \mathrm{~Hz}$. Nevertheless, the amplitude and frequencies selected by this study are within the range utilized by virtually all the studies that used isometric LF exertion to investigate GF-LF coordination in individuals with neurological deficits e.g., [10-12].

\section{Conclusion}

In conclusion, due to the fact that our findings did not indicate any effect of feedback type on GF scaling and GF-LF coupling as well as on the ability to reach the prescribed upper and lower targets, we could claim that the type of feedback presented (i.e., visual and visual plus auditory) does not affect the coordination between GF and LF. However, because tracking tasks are easier to understand and provides additional indices of voluntary force control (e.g., RMSE), we recommend from now on the use of tracking tasks such the one performed in this study during experiments involving isometric LF exertion in externally fixed instrumented objects.

\section{Abbreviations}

GF: Grip force; LF: Load force; $F_{Z}$ : Vertical component of LF; CNS: Central nervous system; bpm: Beats per minute; F/T: Force and torque; AE: Absolute error; GF/LF: Grip force to load force ratio; $r_{\text {max }}$ : Maximum correlation coefficient obtained from the cross-correlation function; ANOVA: Analysis of variance; RMSE: Root mean square error. 


\section{Competing interests}

The authors declare that they have no competing interests.

\section{Authors' contributions}

STP and KCAL were responsible for data collection, processing, and analysis as well as data interpretation and drafting the article. JAB was responsible for drafting the article and revising it critically for scientific method and content. PBF was responsible for conception and design of the study. Also, he was responsible for programing the LabView routines for data acquisition and processing, for data interpretation, and for drafting the article. All authors read and approved the final version of the manuscript.

\section{Acknowledgements}

Authors are thankful to the São Paulo State Research Foundation (FAPESP) for the financial support (FAPESP \#2010/02939-4).

\section{Author details}

${ }^{1}$ Institute of Physical Activity and Sport Sciences and Graduate Program in Human Movement Sciences, Cruzeiro do Sul University, Rua Galvão Bueno 868, $13^{\circ}$ andar, Bloco B, São Paulo 01506-000, SP, Brazil. '2Departament of Physical Education, Bioscience Institute, São Paulo State University at Rio Claro, Av. 24-A, 1515, Bela Vista, Rio Claro, São Paulo, SP 13506-900, Brazil.

Received: 27 March 2012 Accepted: 26 March 2013

Published: 4 April 2013

\section{References}

1. Johansson RS, Westling G: Roles of glabrous skin receptors and sensorimotor memory in automatic control of precision grip when lifting rougher or more slippery objects. Exp Brain Res 1984, 56:550-564.

2. Johansson RS, Westling G: Programmed and triggered actions to rapid load changes during precision grip. Exp Brain Res 1988, 71:72-86.

3. Westling G, Johansson RS: Factors influencing the force control during precision grip. Exp Brain Res 1984, 53:277-284.

4. Blakemore SJ, Goodbody SJ, Wolpert DM: Predicting the consequences of our own actions: the role of sensorimotor context estimation. J Neurosci 1998, 18:7511-7518.

5. Flanagan JR, Wing AM: The stability of precision grip forces during cyclic arm movements with a hand-held load. Exp Brain Res 1995, 105:455-464

6. Jaric S, Collins JJ, Marwaha R, Russell E: Interlimb and within limb force coordination in static bimanual manipulation task. Exp Brain Res 2006, 168:88-97.

7. Flanagan JR, Wing AM: Modulation of grip force with load force during point-to-point arm movements. Exp Brain Res 1993, 95:131-143.

8. Zatsiorsky VM, Gao F, Latash ML: Motor control goes beyond physics: differential effects of gravity and inertia on finger forces during manipulation of hand-held objects. Exp Brain Res 2005, 162:300-308.

9. De Freitas $\mathrm{PB}$, Jaric $\mathrm{S}$ : Force coordination in static manipulation tasks performed using standard and non-standard grasping techniques. Exp Brain Res 2009, 194:605-618.

10. Brandauer B, Timmann D, Hausler A, Hermsdorfer J: Influences of load characteristics on impaired control of grip forces in patients with cerebellar damage. J Neurophysiol 2010, 103:698-708.

11. Krishnan V, De Freitas PB, Jaric S: Impaired object manipulation in mildly involved individuals with multiple sclerosis. Motor Control 2008, 12:3-20.

12. Rost K, Nowak DA, Timmann D, Hermsdorfer J: Preserved and impaired aspects of predictive grip force control in cerebellar patients. Clin Neurophysiol 2005, 116:1405-1414.

13. Hermsdorfer J, Blankenfeld H: Grip force control of predictable external loads. Exp Brain Res 2008, 185:719-728.

14. Uygur M, De Freitas PB, Jaric S: Effects of varying the load force range and frequency on force coordination in static manipulation. Neurosci Letters 2010, 475:115-119.
15. Schmidt RA, Lee TD: Motor control and learning: A behavioral emphasis. 4th edition. Champaign: Human Kinetics; 2005.

16. Sarlegna FR, Baud-Bovy G, Danion F: Delayed visual feedback affects both manual tracking and grip force control when transporting a handheld object. J Neurophysiol 2010, 104:641-653.

17. Miall RC, Jackson JK: Adaptation to visual feedback delays in manual tracking: evidence against the Smith Predictor model of human visually guided action. Exp Brain Res 2006, 172:77-84.

doi:10.1186/1743-0003-10-34

Cite this article as: Pedão et al.: Grip and load force coordination in cyclical isometric manipulation task is not affected by the feedback type. Journal of NeuroEngineering and Rehabilitation 2013 10:34.

\section{Submit your next manuscript to BioMed Central and take full advantage of:}

- Convenient online submission

- Thorough peer review

- No space constraints or color figure charges

- Immediate publication on acceptance

- Inclusion in PubMed, CAS, Scopus and Google Scholar

- Research which is freely available for redistribution

Submit your manuscript at www.biomedcentral.com/submit
C BioMed Central 